\title{
Homogenization of Multi-turn Windings in Electrical Machines with Fewer Number of Conductors
}

\author{
Muhammad Usman Hassan \\ BEAMS Department \\ Université Libre de Bruxelles \\ Brussels, Belgium \\ Muhammad.Hassan@ulb.be
}

\author{
Hugo Carlier \\ BEAMS Department \\ Université Libre de Bruxelles \\ Brussels, Belgium \\ hugo.carlier@ulb.ac.be \\ Yves Mollet \\ BEAMS Department \\ Université Libre de Bruxelles \\ Brussels, Belgium \\ ymollet@ulb.ac.be
}

\author{
Johan Gyselinck \\ BEAMS Department \\ Université Libre de Bruxelles \\ Brussels, Belgium \\ johan.gyselinck@ulb.ac.be
}

\begin{abstract}
AC copper losses are very prominent in high speed electrical machines. Finite element method (FEM) can be used to calculate these losses when modelling each turn of the winding. However, homogenization of windings is generally done to reduce the computational cost of these models. An analytical approach combined with homogenized FEM, is proposed in literature to calculate losses in the frequency domain, considering that each turn is surrounded by a layer of conductors. This paper investigates the accuracy of the approach for lower number of conductors, i.e. when this hypothesis does not hold. For this purpose, results generated by fine and homogenized models are compared.
\end{abstract}

Keywords - proximity losses, homogenization, frequency domain, windings, electrical machines

\section{INTRODUCTION}

Electrical machines are widely being used in automobile, marine and aviation industries quite successfully. Traction applications now demand higher operating frequencies with better torque and power characteristics [3]. The application of hybrid booster for automobiles can reach up to speeds of $20,000 \mathrm{rpm}$ and operating frequency can be raised up to 1.6 $\mathrm{kHz}$. At these frequencies and speeds, electrical machines are prone to AC copper losses and they can significantly affect the machine performance characteristics [4].

Making fine models in a 2-D and 3D FE model is too computationally expensive for it to be practical. That is why homogenization is the way forward. Meeker et al [5] gives analytical formulas for calculation skin and proximity effects for hexagonally packed wires but it works more accurately for lower frequencies. Moreau et al. [6] explains the use of a frequency dependent, complex magnet permeability model for rectangular conductors in transformer windings.
Podoltsev et al. [7] assumes round wires packed in square grids for transformer windings. Numerical solutions for complex permeability for various fills are graphically presented. Rossmanith [8] and Nan, Xi and Sullivan et al. [9] give analytical formulas for proximity effect losses in hexagonally packed windings. For round wires with hexagonal packing Gyselinck et al. [2] proposed numerical method which effectively covers proximity and skin effect. Niyomsatian et al. [10] implemented frequency domain homogenization for litz wire bundle on 2-D translationalsymmetry transformer model and 3-D and the axisymmetric inductor model respectively.

All these methods are primarily used for transformer design in order to compute proximity effects in the windings.

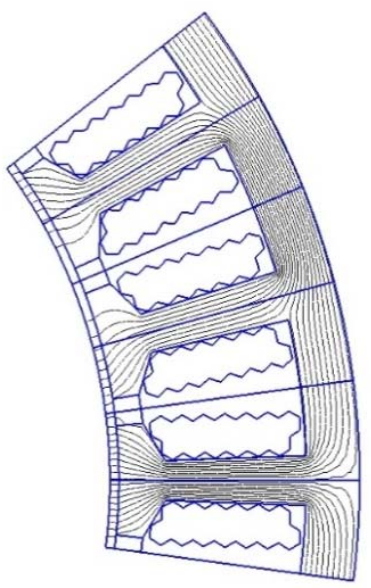

(a)

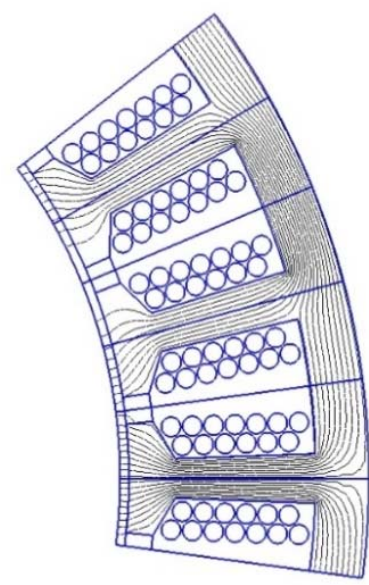

(b)
Fig. 1. (a) Homogenized model of stator with flux lines (b) fine model of stator with flux lines 
However, Gyselinck's work is already extended for electrical machines with form windings in [1] which shows the ease of implementation of frequency domain homogenization in electrical machines.

This approach is very successful for higher number of conductors because it considers that each turn is surrounded by a layer of conductors. In this paper, the homogenization technique proposed in [2] is implemented on stator windings with fewer number of round conductors. i.e. when each turn cannot be considered as surrounded by a layer of conductors. A homogenized model is proposed and compared with fine model which are shown in Figure 1 (a) and (b) respectively.

This paper focuses on proximity losses calculation by frequency domain homogenization. The results obtained from the two models are compared to observe the accuracy of the homogenization technique.

\section{GEOMETERY OF StATOR SLOTS AND CONDUCTORS}

In order to study the losses, $1 / 8$ th of a machine crosssection with 26 conductors in each slot is considered. Only one pole of the stator has been modelled to simplify the geometry as shown in Figure 1. The homogenized model Figure 1 (a) has windings placed in the hexagonal network of cells. The geometry of a single slot is represented in Figure 2 and the corresponding parameters are given in Table 1.

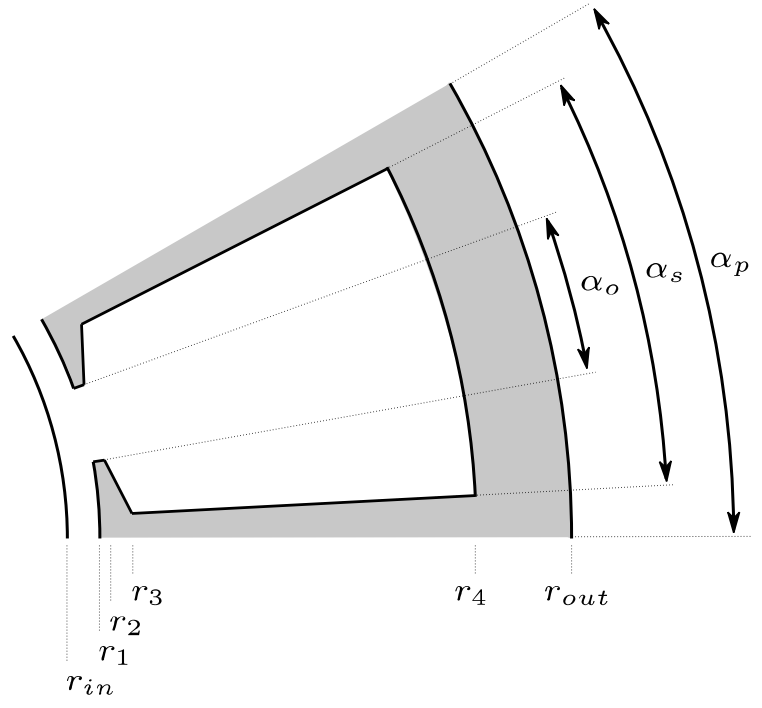

Fig. 2. Parametrized geometry of a slot

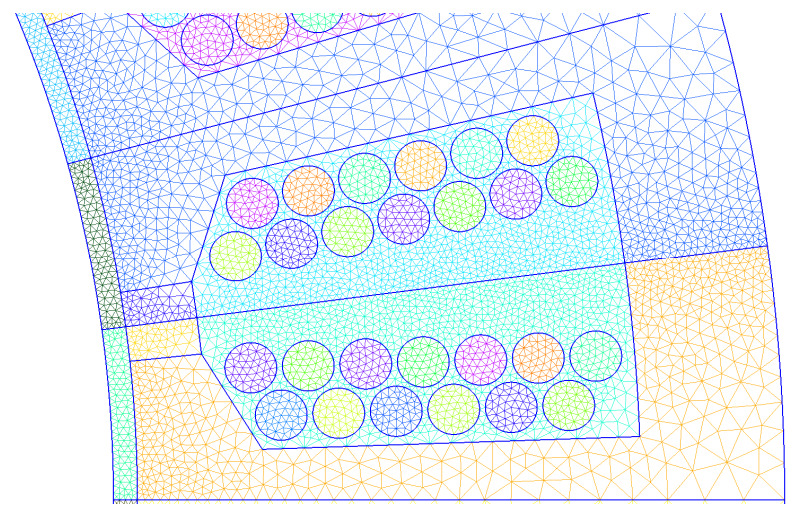

Fig. 3. Mesh of fine model
TABLE I. Geometrical Dimensions of the SLOT

\begin{tabular}{|c|c|}
\hline Parameters & Value \\
\hline $\mathrm{r}_{\text {in }}$ & $18.66 \mathrm{~mm}$ \\
\hline $\mathrm{r}_{1}$ & $19 \mathrm{~mm}$ \\
\hline $\mathrm{r}_{2}$ & $20 \mathrm{~mm}$ \\
\hline $\mathrm{r}_{3}$ & $20.75 \mathrm{~mm}$ \\
\hline $\mathrm{r}_{4}$ & $26 \mathrm{~mm}$ \\
\hline $\mathrm{r}_{\text {out }}$ & $28 \mathrm{~mm}$ \\
\hline$\alpha_{\mathrm{o}}$ & $5^{\circ}$ \\
\hline$\alpha_{\mathrm{s}}$ & $11^{\circ}$ \\
\hline$\alpha_{\mathrm{p}}$ & $15^{\circ}$ \\
\hline
\end{tabular}

The mesh (as shown in Figure 3) is kept fine around the air gap and conductors in the slot for fine model in order to generate accurate results. The construction of geometry and corresponding FE calculations on the proposed meshes are carried out by using the open-source ONELAB bundle software [11]

A sinusoidal current (in space and in time) is imposed in the current layer, which is situated on the surface of the rotor to emulate the effect of rotating magnetic field as produced by permanent magnets. A current of amplitude 250 A-turns is considered in the current layer.

The imposed current in the current layer is selected in such a way that in open circuit conditions the induction in the middle of the tooth is around 1 Tesla to remain in the linear part of the BH curve of the magnetic flux. Figure 4 shows the distribution of norm of magnetic flux density in the stator iron. The current layer saves computational time as it does not require to model the rotor geometry.
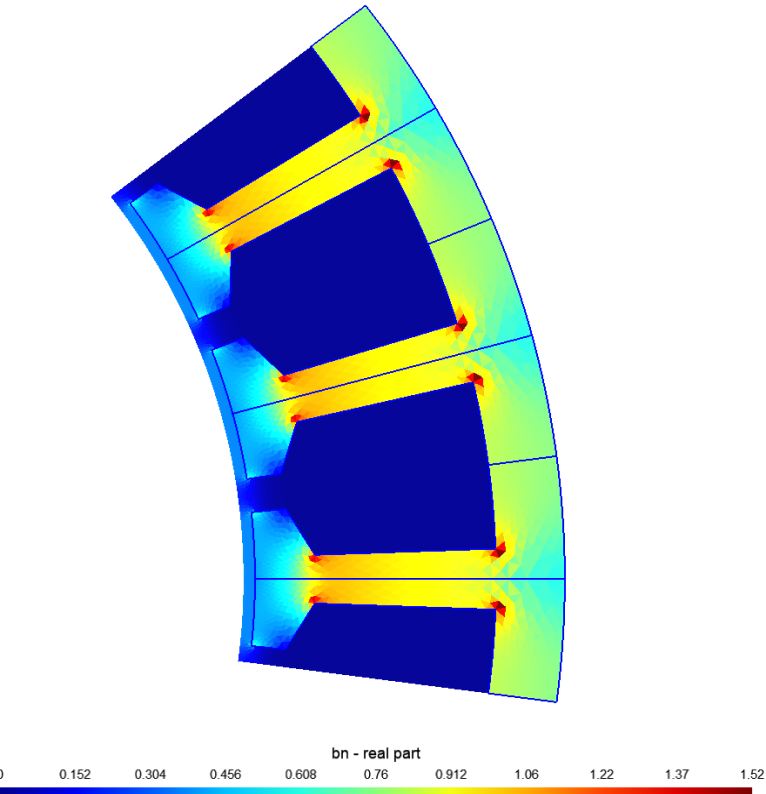

Fig. 4. An illustration of norm of sinusoidal magnetic flux density in the stator iron having imposed amplitude of $1 \mathrm{~T}$ 


\section{Proximity EFFECT In CONDUCTORS}

\section{A. Definitions and notations}

A frequency $f=\omega / 2 \pi$ is assumed throughout this paper. For fine model, the conductors in the slots are modelled as massive conductors each having conductivity of copper as $\sigma=60 \mathrm{MS} / \mathrm{m}$ with radius $r=0.36 \mathrm{~mm}$. The permeability of conductors is taken as $\mu_{0}=4 \pi \cdot 10^{-7} \mathrm{H} / \mathrm{m}$. The net current imposed in the conductors is zero (open-circuit case).

For homogenized model, the induced currents are not computed instead the whole area is modelled as stranded conductors in which uniform current density is adopted. The fill factor of the winding is $\lambda=0.734$ (which is the ratio between area of the wire and area of the hexagonal packing of the conductor). The relative permeability of stator iron is taken as $\mu_{r}=6631$ considering the linear part of the characteristic curve give in [12].

The skin depth is calculated using $\delta=\sqrt{\frac{2}{\sigma \omega \mu_{0}}}$ from here reduced frequency is obtained using factor $d=r / \delta[13]$.

\section{B. Frequency domain calculations for fine model}

The frequency domain calculations are carried out, for fine model (as shown in Figure 1 (b)), with adequate current and necessary boundary conditions. The active power $\mathrm{P}$ is calculated from the local current density $j[2]$ :

$$
P=l \int_{\Omega} \frac{j^{2}}{\sigma} d \Omega
$$

where $l$ is the length and $\Omega$ is the cross-sectional area of the conductors. The $j^{2}=\boldsymbol{j} \cdot \boldsymbol{j}^{*} / 2$ is the r.m.s. value of current density. The net current imposed in the conductors is zero.

For massive conductors, the induce current is calculated as [14]:

$$
j=\sigma\left(-\frac{\partial A}{\partial t}-\nabla \cdot V\right)
$$

where $A$ is the magnetic vector potential and $V$ is electric scalar potential.

\section{Frequency domain calucations for homogenized model}

For homogenized model, the proximity losses can be computed from the induction field crossing the coil region [2]:

$$
P_{B}=p_{B}(d) \frac{\pi}{4} \sigma l r^{4} \omega^{2} \int_{\Omega} b_{e}^{2} d \Omega
$$

where the magnetic field density $b_{e}^{2}=\boldsymbol{b}_{\boldsymbol{e}} \cdot \boldsymbol{b}_{\boldsymbol{e}}{ }^{*} / 2$ is crossing the cross-sectional area of the coil region $\Omega$.

The dimensionless factor $p_{B}$ is given as [2]:

$$
p_{B}(d)=\frac{4}{d^{2}} \operatorname{Im}\left(\frac{J_{2}((1+i) d)}{J_{0}((1+i) d)}\right)
$$

where $\boldsymbol{J}_{\boldsymbol{k}}$ is the first kind Bessel function with order $k$.

For low frequencies $d$ tends to 0 and $p_{B}$ tends to 1 .

\section{RESULTS}

From (1) and from (3), the proximity losses are calculated for the fine model and homogenized model respectively. The proximity losses are calculated in both models at reduced frequency $d=0.05$ is shown in Figures 5 and 6 respectively. This shows that losses are concentrated near the opening of the slots.

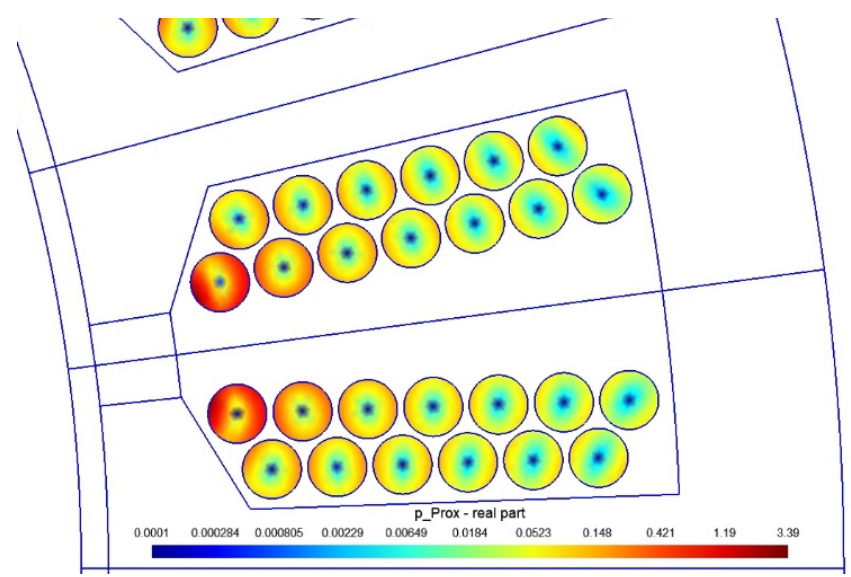

Fig. 5. Proximity losses $(\mathrm{W} / \mathrm{m})$ in fine model at reduced frequency 0.05

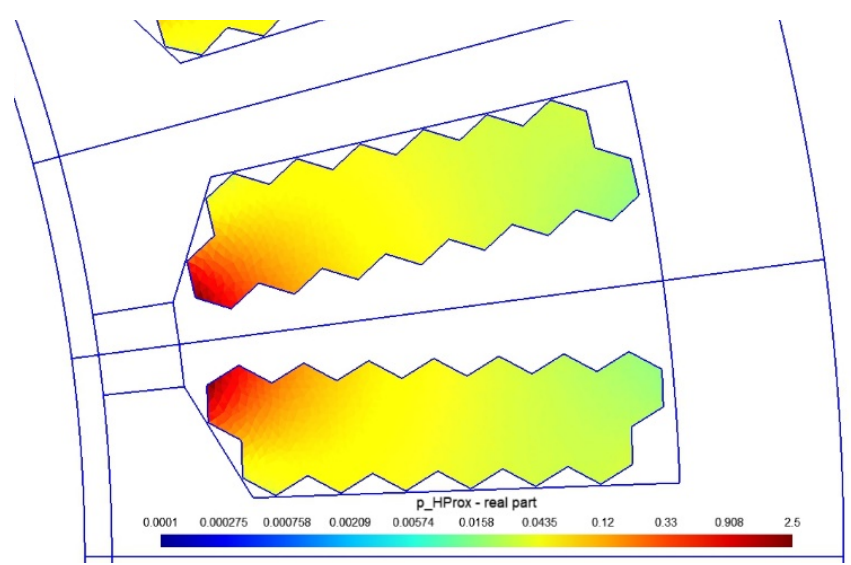

Fig. 6. Proximity losses $(\mathrm{W} / \mathrm{m})$ in homogenized model at reduced frequency 0.05

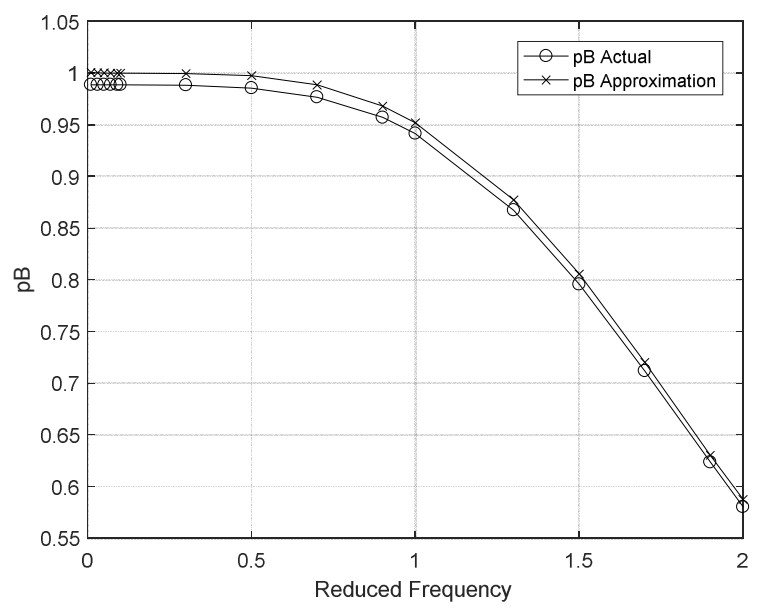

Fig. 7. Dimensionless coefficent for proximity losses with respect to reduced frequency 


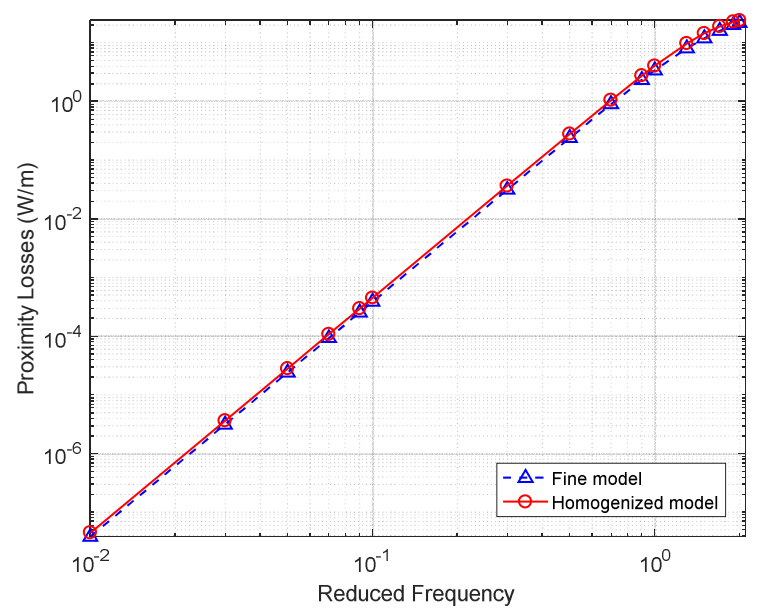

Fig. 8. Proximity losses for fine model and homogenized model with respect to reduced frequency

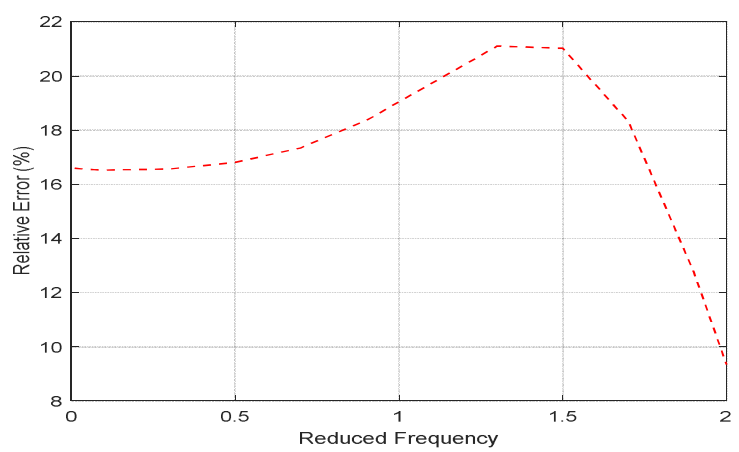

Fig. 9. Relative error between losses calculated from fine model and homogenized model

\section{A. Proximity loss approximation for homogenized model}

The dimensionless coefficient for proximity effect $p_{\mathrm{B}}$ was calculated for fine model using (3) as shown in Figure 7. The actual value of $p_{\mathrm{B}}$ follows the similar pattern for round conductors as described in [2] which is calculated for larger number of conductors in a slot. However, the error between the curves increases from $3 \%$ to $30 \%$ for reduced frequencies of 1 to 2 . The value of $p_{\mathrm{B}}$ is normalized to 1 so that a good agreement with the results in [2] can be obtained. Using $5^{\text {th }}$ order polynomial given below, an approximation of $p_{\mathrm{B}}$ is calculated, which is given as:

$$
\begin{gathered}
p_{B}^{\prime}(d)=0.024 d^{5}-0.063 d^{4}-0.042 d^{3}+0.043 d^{2} \\
-0.01 d+1
\end{gathered}
$$

The fifth order polynomial gives an acceptable fit and reduces the relative error observed in Figure 9. Using (5) in (3), the proximity losses in homogenized model are calculated. The losses in fine model are also calculated using (1) and both results are plotted in Figure 8. It can be observed that the two curves are in a good agreement with each other.

For reduced frequencies $0<d<1$ the approximate value of $p_{\mathrm{B}}$ and $p_{\mathrm{B}}$ in [2] are aproaching to 1 and can be approximated to 1 , as there is less than $5 \%$ error between them. This shows that by reducing the number of conductors the homogenization technique still holds. For greater values of reduced frequencies $(d>1), p_{\mathrm{B}}$ curve should be obtained to calucalte losses. The maximum relative error is $21 \%$ for reduced frequency of $(1.25<d<1.5)$ as it can be seen in Figure 9.

\section{CONCLUSIONS}

For electrical machines with fewer number of round conductors in their windings, the frequency domain homogenization method from [2] is valid for reduced frequency values $0<d<1$. For $d>1$, a $p_{\mathrm{B}}$ curve should be obtained specific to the geometrical model. This method can have a better impact if the model is refined to obtain proximity losses influenced by the reactive power consumed by the machine.

\section{ACKNOWLEDGMENT}

This paper is part of the European Industrial Doctorate on Next Generation for sustaINable auTomotive ElectRical ACtuaTion (INTERACT) project which has received funding from the European Union Horizon 2020 research and innovation programme under grant agreement No 766180. Muhammad Usman Hassan is an Early Stage Researcher on this project.

\section{REFERENCES}

[1] Gyselinck Johan, Patrick Dular, Nelson Sadowski, Patrick Kuo-Peng, and Ruth V. Sabariego. "Homogenization of form-wound windings in frequency and time domain finite-element modeling of electrical machines." IEEE trans. on magnetics 46, no. 8 (2010): 2852-2855.

[2] Gyselinck, Johan, and Patrick Dular. "Frequency-domain homogenization of bundles of wires in 2-D magnetodynamic FE calculations." IEEE trans. on magnetics 41, no. 5 (2005): 1416-1419.

[3] Tangudu, Jagadeesh K., and Thomas M. Jahns. "Comparison of interior PM machines with concentrated and distributed stator windings for traction applications." In 2011 IEEE Vehicle Power and Propulsion Conference, pp. 1-8. IEEE, 2011.

[4] Mellor, Phil, Rafal Wrobel, and Nick Simpson. "AC losses in high frequency electrical machine windings formed from large section conductors." in IEEE Energy Conversion Congress and Exposition (ECCE), pp. 5563-5570, 2014.

[5] Meeker, David C. "An improved continuum skin and proximity effect model for hexagonally packed wires." Journal of Computational and Applied Mathematics 236, no. 18 (2012): 4635-4644.

[6] Moreau, O., L. Popiel, and J. L. Pages. "Proximity losses computation with a 2D complex permeability modelling." IEEE Transactions on Magnetics 34, no. 5 (1998): 3616-3619.

[7] Podoltsev, Alexander D., Irina N. Kucheryavaya, and Boris B. Lebedev. "Analysis of effective resistance and eddy-current losses in multiturn winding of high-frequency magnetic components." IEEE Transactions on Magnetics 39, no. 1 (2003): 539-548.

[8] Rossmanith, Hans, Manfred Albach, Janina Fischer, and Alexander Stadler. "Improved characterization of the magnetic properties of hexagonally packed wires." EPE Journal 22, no. 4 (2012): 5-10.

[9] Nan, Xi, and Charles R. Sullivan. "An equivalent complex permeability model for litz-wire windings." In Fourtieth IAS Annual Meeting. Conf. Record of the 2005 Industry Applications Conference, 2005., vol. 3, pp. 2229-2235. IEEE, 2005.M. Young, The Technical Writer's Handbook. Mill Valley, CA: University Science, 1989.

[10] Niyomsatian, Korawich, J. Van den Keybus, R. V. Sabariego, and Johan Gyselinck. "Frequency-domain homogenization for litz-wire bundles in finite element calculations." in 18th European Conference on Power Electronics and Applications, pp. 1-10. IEEE, 2016.

[11] Open Numerical Engineering LABoratory, http://openlab.info/.

[12] Rashid, Noor Ashikin Mohd, and WANNORLW MAHADI. "Simulation and Statistical Approaches on Electrical Steel's Magnetic Behaviour under Unidirectional Single Sheet Tester." Sains Malaysiana 43, no. 6 (2014): 909-914.

[13] Robert Frédéric, Pierre Mathys, and J-P. Schauwers. "Ohmic losses calculation in SMPS transformers: numerical study of Dowell's approach accuracy." IEEE transactions on magnetics 34, no. 4 (1998): 1255-1257.

[14] Chari M., and Z1 Csendes. "Finite element analysis of the skin effect in current carrying conductors." IEEE trans. on magnetics 13 , no. 5 (1977): 1125-1127. 